Iyo Alexis Cruz-Piza; Jefferson Andrés Pozo-Lucio; Víctor Leonel Gómez-Naranjo

http://dx.doi.org/10.35381/racji.v5i3.1089

\title{
El estado de embriaguez y su incidencia en los accidentes de tránsito
}

The state of intoxication and its incidence in traffic accidents

\author{
lyo Alexis Cruz-Piza \\ ub.iyocruz@uniandes.edu.ec \\ Universidad Regional Autónoma de los Andes, Babahoyo \\ Ecuador \\ https://orcid.org/0000-0002-2655-172X \\ Jefferson Andrés Pozo-Lucio \\ jeffersonandrespozolucio03@gmail.com \\ Universidad Regional Autónoma de los Andes, Babahoyo \\ Ecuador \\ Víctor Leonel Gómez-Naranjo \\ victorgomez618@gmail.com \\ Universidad Regional Autónoma de los Andes, Babahoyo \\ Ecuador
}

Recibido: 31 de octubre de 2020

Revisado: 10 de octubre de 2020

Aprobado: 05 de diciembre de 2020

Publicado: 10 de diciembre de 2020 
Iyo Alexis Cruz-Piza; Jefferson Andrés Pozo-Lucio; Víctor Leonel Gómez-Naranjo

\title{
RESUMEN
}

El alcohol, es uno de los trascendentales elementos que contribuyen a las contravenciones de tránsito en Ecuador y el mundo, principalmente ocasionando lesiones y muertes. El objetivo de esta investigación es fundamentar jurídica y doctrinariamente el significado de los accidentes de tránsito a causa del estado de embriaguez de los conductores. Se realizó una revisión de las normas y estadísticas de los hechos ocasionados por este flagelo. Se apoya la presente indagación en la descripción documental que contribuye al análisis de diversas entidades observables en la realidad. Con un diseño bibliográfico contribuyó. Así mismo se empleó el método analítico sintético nos permite analizar los fenómenos y causas. Se obtuvo una de muestra de 21 personas, a los cuales se les administró un cuestionario. En conclusión, los encuestados ratifican que la mayor parte de accidentes de tránsito se dan por ingerir alcohol, y no existe control por parte de los agentes de tránsito.

Descriptores: Adicción; mortalidad; educación antialcohólica. (Palabras tomadas del Tesauro UNESCO).

\begin{abstract}
Alcohol is one of the transcendental elements that contribute to traffic offenses in Ecuador and the world, mainly causing injuries and deaths. The objective of this research is to legally and doctrinally substantiate the meaning of traffic accidents due to the drunken state of drivers. A review of the rules and statistics of the events caused by this scourge was carried out. The present inquiry is based on the documentary description that contributes to the analysis of various observable entities in reality. With a bibliographic design he contributed. Likewise, the synthetic analytical method was used, allowing us to analyze the phenomena and causes. A sample of 21 people was obtained, to whom a questionnaire was administered. In conclusion, the respondents confirm that the majority of traffic accidents are caused by drinking alcohol, and there is no control by traffic officers.
\end{abstract}

Descriptors: Addiction; mortality; anti-alcoholic education. (Words taken from the UNESCO Thesaurus). 
lustitia Socialis. Revista Arbitrada de Ciencias Jurídicas.

Año V. Vol. V. №3. Edición Especial. 2020-III:

Universidad Regional Autónoma de los Andes

Hecho el depósito de Ley: FA2016000064

ISSN: 2542-3371

FUNDACIÓN KOINONIA (F.K). Santa Ana de Coro, Venezuela

Iyo Alexis Cruz-Piza; Jefferson Andrés Pozo-Lucio; Víctor Leonel Gómez-Naranjo

\section{INTRODUCCIÓN}

Esta investigación aborda un problema social que perjudica y afecta a la población; física, psíquica y económicamente por las personas involucradas, y también a la seguridad ciudadana en general, en muchas ocasiones, a bienes y propiedades del Estado. Es importante entender el fenómeno de los accidentes de tránsito, aportar en la prevención y disminución de los mismos. Es decir, se convierte en un problema prioritario de salud pública, en virtud del aumento de la tasa de mortalidad, según las estimaciones del informe sobre la situación mundial en seguridad vial de la Organización Mundial de la Salud, publicado en el 2015, Ecuador ocupa el séptimo puesto en Latinoamérica en tasa de fallecimientos por accidentes de tránsito y el décimo tercero a escala, global. (Algora-Buenafé y otros, p.54)

Fuera de las fronteras, en Chile, la ley relacionada a las sanciones por ingesta de alcohol, supone, es decir, presume que estos estados comprometen seriamente la seguridad de quienes se incorporan al sistema del tránsito, de modo que, no puedan circular sin ver potencialmente afectadas su vida, su salud o su propiedad. Por otro lado, el compromiso es tan serio que requiere de una sanción penal, es decir, requiere ser considerado delito, pues ningún otro medio extrajurídico o de alguna de las restantes ramas del Derecho es suficiente para resguardar adecuadamente este objeto de tutela. (Cabeza-Cabezas, 2010).

Continuando en nuestra Nación, existe un alto índice de accidentes de tránsito provocado por los conductores en estado de embriaguez, la falta de recursos, control y de educación provoca que los conductores se encuentren involucrados en los accidentes de tránsito, estos no miden el peligro en los que se encuentran vinculados al conducir un vehículo en estado de embriaguez, tampoco establecen la problemática que lleva consigo, y a quienes afectaría su impericia, no toman en cuenta que no solo pueden ser ellos los afectados sino que también a las personas que los rodean y a sus familiares.

El índice de accidentes de tránsito va aumentando debido a que no existe el control suficiente por parte de las autoridades del sector. Las sanciones por conducir un vehiculó en estado de embriaguez debe ser más drásticas para disminuir los 
accidentes de tránsito ya que muchos de los conductores recapacitarían y así dejaran de conducir un vehículo en ese estado. Sin embargo, con la entrada en vigencia del Código Orgánico Integral Penal (COIP) aporta consigo la ardua tarea de afrontar los impresionantes índices de accidentes de tránsito que ocurren en el país, sobre todo aquellos que lesionan los bienes legales relacionados al ser humano, como son la vida y la integridad de las personas. (Duran,2014).

Pero se evidencia una falta de interés en las sanciones que deberían ser más contundentes con el objeto de crear conciencia, en concordancia con lo que afirma Andrade-Torres y otros (2020):

un manejo poco prolijo en el ejercicio de la legislación nacional, así como en la emisión de actos normativos y administrativos ha resultado en la excesiva, dispersa, desordenada y desarticulada formulación de normativa, y ello ha permitido que los entes llamados a velar por la armonía del ordenamiento jurídico tengan serios problemas al ejecutar sus funciones, y ello ha afectado seriamente en la administración de justicia en última instancia, por razones que a primera vista resultan obvias. (p.146)

Las sanciones que se reciben por conducir un vehículo en estado de embriaguez, están claramente tipificadas en la Ley Orgánica de Transporte Terrestre, Tránsito y Seguridad Vial. En este sentido los artículos; 376 y 385 establecen los siguiente:

Artículo 376.- Muerte causada por conductor en estado de embriaguez o bajo los efectos de sustancias estupefacientes, psicotrópicas o preparados que las contengan. - La persona que conduzca un vehículo a motor en estado de embriaguez o bajo los efectos de sustancias estupefacientes, psicotrópicas o preparados que las contengan y ocasione un accidente de tránsito del que resulten muertas una o más personas.

Sanción: Pena privativa de libertad de diez a doce años, Revocatoria definitiva de la licencia para conducir vehículos

Articulo 385.-Conducción de vehículo en estado de embriaguez. - La persona que conduzca un vehículo en estado de embriaguez, será sancionada de acuerdo con la siguiente escala:

1. Si el nivel de alcohol por litro de sangre es de 0,3 a 0,8 gramos, se aplicará multa de un salario básico unificado del trabajador en general, pérdida de cinco puntos en su licencia de conducir y cinco días de privación de libertad. 
2. Si el nivel de alcohol por litro de sangre es mayor de 0,8 hasta 1,2 gramos, se aplicará multa de dos salarios básicos unificados del trabajador en general, pérdida de diez puntos en su licencia de conducir y quince días de privación de libertad.

3. Si el nivel de alcohol por litro de sangre supera 1,2 gramos, se aplicará multa de tres salarios básicos unificados del trabajador en general, la suspensión de la licencia por sesenta días y treinta días de privación de libertad.

Para las o los conductores de vehículos de transporte público liviano o pesado, comercial o de carga, la tolerancia al consumo de cualquier sustancia estupefaciente, psicotrópica o preparado que las contengan es cero, y un nivel máximo de alcohol de 0,1 gramos por cada litro de sangre.

En caso de exceder dicho límite, la sanción para el responsable será, pérdida de treinta puntos en su licencia de conducir y pena privativa de libertad de noventa días. Además, en todos estos casos, como medida preventiva se aprehenderá el vehículo por veinticuatro horas.

En el sistema penitenciario, la mayor población es precisamente la relacionada con drogas: 11000 presos recluidos en 36 centros de rehabilitación que operan en el país. Sin embargo, en su trabajo de investigación Vasco-Intriago (2017) afirma, sorpresivamente, morir por un accidente de tránsito, no es sancionado como debería y a parte, el culpable es premiado con la sustitución de su pena, lo que ha generado una inseguridad jurídica para la víctima, al no poder recibir una reparación integral digna de sus derechos. (p.9). Lo cual coloca en gran riesgo la vida de los ciudadanos, que se ven vulnerado sus derechos por la débil aplicación de la ley en esta materia que nos ocupa.

Por otro lado, de acuerdo al anuario estadístico de transporte 2018, la principal causa de siniestros fue la impericia e imprudencia del conductor, con un total de 12.660 siniestros, que representan el 49,6\%; seguido de exceso de velocidad con 4.068 siniestros, con el $15,9 \%$ del total de siniestros de tránsito nacionales. Por embriaguez o droga el $7.5 \%$. 


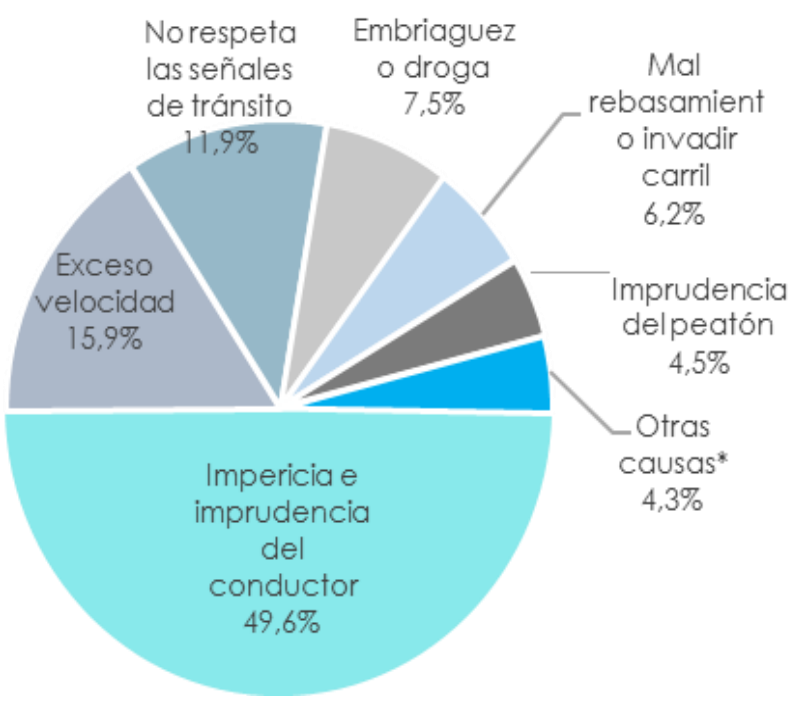

\section{Gráfico 1.}

Fuente: Agencia Nacional de Tránsito (2018).

En el mismo boletín se define lo siguiente Embriaguez o droga: como la pérdida transitoria o manifiesta disminución de las facultades físicas y mentales normales, ocasionadas por el consumo de bebidas alcohólicas o estupefacientes, respectivamente, y que disminuye las capacidades para conducir cualquier tipo de vehículo (ANT, 2012). Lo que califica como un alto riesgo, tanto para el conductor como para los ciudadanos, y requiere estrategias y acciones contundentes que permitan la disminución de las altas cifras de siniestralidad. Los siguientes autores relacionan los delitos de transito de en función del COIP, Granda-Torres \& Del CisneHerrera (2019), como los delitos contra la responsabilidad ciudadana, delitos contra la estructura del estado constitucional, delitos de terrorismo e infracciones de tránsito. Además, como delitos contra el derecho del buen vivir y delitos contra el ambiente y la naturaleza o Pacha Mama, se encuentran los delitos que atentan a la salud, recordemos que guarda relación con los índices de mortalidad y salud pública.

En sentido se plantea la siguiente interrogante ¿Cómo disminuir los accidentes de tránsito por parte de los conductores de vehículos a causa del estado de embriaguez en el recinto Mata De Cacao? El objetivo de esta investigación es fundamentar jurídica 
y doctrinariamente el significado de los accidentes de tránsito a causa del estado de embriaguez de los conductores de vehículos.

\section{METODOLOGÍA}

Para la metodología aplicada, se apoya la presente investigación en la descripción documental, según Hernández Sampieri, Fernández Collado \& Baptista (2014), es de característica investigativa que contribuye al análisis de diversas entidades observables en la realidad. Con un diseño bibliográfico contribuyó a la recolección de datos relacionado al estudio. Así mismo se empleó el método analítico sintético, se considera a este como un procedimiento fundamental de toda investigación debido a que este nos permite analizar los fenómenos y causas de los problemas. Este método es de suma importancia para nuestro tema de revisión debido a que gracias a este hemos podido analizar las causas y consecuencias del alcoholismo en los conductores de vehículos de este sector para poder realizar una síntesis compleja de este fenómeno. Siendo la población moderadamente grande, la muestra se determinó tomando en consideración a los conductores de vehículos del recinto con una cifra aproximada de 400 conductores, aplicando la formula correspondiente obteniendo una de muestra de 21 personas, a los cuales se les administró un cuestionario.

\section{RESULTADOS}

De acuerdo a la encuesta aplicada se demuestra que un $62 \%$ de la población considera que el principal motivo para conducir un vehículo es la necesidad, mientras que un $24 \%$ indica que lo realiza por paseo y un $14 \%$ lo realiza por pasatiempo; un $52 \%$ de la población no ha conducido en estado de embriaguez, mientras que un $29 \%$ lo ha realizado pocas veces y un $19 \%$ definitivamente no ha conducido en estado de embriaguez; el 62\% de la población afirma que una persona que se encuentra en estado de embriaguez no tiene suficiente capacidad para conducir mientras que un $19 \%$ estuvo de acuerdo que una persona que se encuentra en estado de embriaguez si tiene suficiente capacidad para conducir; un $71 \%$ de la población considera que el 
mayor número de accidentes de tránsito en nuestro país es causado por el estado de embriaguez de los conductores mientras que un 10\% considera que la causa de los accidentes de tránsito no es debido al estado de embriaguez; en base a la encuesta realizada se llegó a la conclusión de que casi siempre se producen los accidentes de tránsito en este recinto con un porcentaje de $52 \%$; un $76 \%$ de la población no conoce el Art. 386 del código orgánico integral penal (COIP) que habla acerca de la contravención de transito por conducir un vehículo en estado de embriaguez esto quiere decir que existe un alto grado de desconocimiento de esta ley y se concluye que un $76 \%$ de la población afirma que es necesario incrementar controles sorpresivos a los señores conductores con la prueba de alcoholemia mientras que un $24 \%$ considera que posiblemente se deba realizar estos controles.

\section{DISCUSIÓN}

Los resultados de este trabajo muestran un problema social y prioritario por fallecimiento en accidentes de tránsito ocasionado por conductores en estado de embriaguez, que afecta a la población ecuatoriana tanto jóvenes como mayares. Se requiere establecer estrategias y aplicación más efectiva de las normas para garantizar la seguridad vial de los ciudadanos evitando los acontecimientos inseguros de los conductores, el exceso de velocidad, alta ingesta de alcohol, siendo un alto peligro para los grupos vulnerables los peatones.

\section{CONCLUSIONES}

Los conductores y los agentes de tránsito si conocen los efectos negativos que produce el alcohol, pero el problema mayor es la adicción que tienen las personas al ingerir este producto.

Los conductores conocen los tipos de alcohol permitidos por la ley ecuatoriana, pero es el irrespeto a la ley COIP (consumo de alcohol) el causante principal de accidentes de tránsito.

Las personas especialmente del sexo masculino manifiestan que el alcohol es la solución inmediata y más efectiva a la depresión, problemas psicológicos y afectivos. 
Los encuestados ratifican que la mayor parte de accidentes de tránsito se dan por ingerir alcohol, y no existe control por parte de los agentes de tránsito.

\section{FINANCIAMIENTO}

No monetario.

\section{AGRADECIMIENTO}

A la Universidad Regional Autónoma de Los Andes; por motivar el desarrollo de la Investigación.

\section{REFERENCIAS CONSULTADAS}

Agencia Nacional de Tránsito. Estadísticas de transporte terrestre y seguridad vial, Ecuador. ANT (2016). [National Transit Agency. Statistics on land transport and road safety, Ecuador. ANT 2016]. Disponible en: https://n9.cl/d3p6

Andrade-Torres, J., Erazo-Álvarez, J., Trelles-Vicuña, D., \& Narváez-Zurita, C. (2020). Conciliación en asuntos relacionados con infracciones de tránsito en el Ecuador Vulneración de derechos fundamentales. [Conciliation in matters related to traffic violations in Ecuador Violation of fundamental rights]. IUSTITIA SOCIALIS, 5(8), 142-161. http://dx.doi.org/10.35381/racji.v5i8.566

Algora-Buenafé AF, Russo-Puga M, Suasnavas-Bermúdez PR, Merino-Salazar P, Gómez-García AR. (2017). Tendencias de los accidentes de tránsito en Ecuador: 2000-2015. [Trends in road accidents in Ecuador: 2000-2015]. Rev Gerenc Polít Salud. 16 (33): 52-58. https://doi.org/10.11144/Javeriana.rgps1633.tate

Asamblea Nacional del Ecuador. (2014). Código Orgánico Integral Penal. [ Comprehensive Criminal Organic Code]. Quito: Registro Oficial.

Cabezas-Cabezas, C. (2010). Los delitos de conducción bajo la ingesta de alcohol o sustancias estupefacientes como delitos de peligro. [Driving Offences under Alcohol Influence or Mood Altering Substances as Crimes of Endangerment] Revista de Derecho de la Pontificia Universidad Católica de Valparaíso. 34, 227-280. http://dx.doi.org/10.4067/S0718-68512010000100007

Dirección Nacional de Control del Tránsito y Seguridad Vial de la Policía Nacional del Ecuador. (2019). Base de Datos de Personas, Vía y Vehículos 2015-2019. https://n9.cl/tinv 
Duran, P. (2014). La ley de tránsito desde el punto de vista criminológico. [The law of transit from a criminological point of view]. Boletín criminológico Fiscalía General del Estado-Ecuador.

Granda-Torres, G., Herrera-Abrahán, C. (2020). Análisis de los tipos penales y su importancia para determinar responsabilidad penal. [Analysis of criminal types and their importance in determining criminal responsibility]. IUSTITIA SOCIALIS, 5(7), 220-232. http://dx.doi.org/10.35381/racji.v4i7.443

Hernández Sampieri, R., Fernández Collado, C., \& Baptista, L. (2014). Metodología de la investigación. [Research methodology]. México: Editorial Mc - Graw - Hill Interamericana. Sexta edición.

Instituto Nacional de Estadística y Censos. Población y Demografía. (2018). Boletín técnico $\mathrm{N}^{\circ}$ 01-2019-transporte. Anuario de estadísticas de transporte. [Technical Bulletin No. 01-2019-transport. Yearbook of transport statistics]. Recuperado: http://www.ecuadorencifras.gob.ec

Ley Orgánica Reformatoria a la Ley Orgánica de Transporte Terrestre, Tránsito y Seguridad Vial. (2014). [Organic Law Reforming the Organic Law on Land Transport, Transit and Road Safety]. Registro Oficial Suplemento 407. Ecuador.

Vasco Intriago, A. (2017). La muerte en delitos de tránsito y la suspensión condicional de la pena. [Death in traffic offences and conditional suspension of sentence]. Trabajo de grado. Universidad Técnica de Ambato. Facultad de Jurisprudencia y Ciencias Sociales. Carrera de Derecho. 